\title{
Agroconnect - Sistema de Apoio à Tomada de Decisão de Tecnologiasem Fio de Transmissão de Dados para Soluções de IoT no Agronegócio
}

Cleberson Barbosa das Chagas - Universidade Federal Rural de Pernambuco

(ORCID - 0000-0003-3805-4904) - clebrsonn@gmail.com

Fernando Antônio Aires Lins - Universidade Federal Rural de Pernambuco

(ORCID - 0000-0002-4007-3891) - fernandoaires@ufrpe.br

Obionor de Oliveira Nóbrega - Universidade Federal Rural de Pernambuco

(ORCID - 0000-0003-1721-9669) - obionor.nobrega@ufrpe.br

\begin{abstract}
Resumo - O agronegócio no Brasil se diferencia de outros mercados pelo grande investimento em soluções tecnológicas que possibilitem maior eficiência na cadeia de produção. Dentre as tecnologias mais investidas pode-se destacar a Internet das Coisas (IoT) que permite sentir e atuar em tempo real. Neste contexto, identificar qual tecnologiade transmissão de dados mais se adequa a uma determinada solução IoT é um problema relevante, pois a seleção equivocada da tecnologia de transmissão de dados em projeto deIoT pode resultar em soluções inadequadas ou ineficientes para o agronegócio, visto queexistem diferentes cenários, com áreas geográficas distintas, culturas e/ou cultivos variados. Este trabalho tem como objetivo apresentar um sistema de apoio à tomada de decisão para a seleção de tecnologias de transmissão sem fío em projetos de IoT no agronegócio.
\end{abstract}

Palavras-chave: Internet das Coisas, Agronegócio, tecnologia de transmissão sem Fio

\section{Agroconnect - Wireless Data Transmission Technology Decision Support System for IoT in Agribusiness}

\begin{abstract}
Agribusiness in Brazil has differentiated itself from other markets due to thelarge investment in technological solutions that enable greater efficiency in its entire production chain. Among the technologies that have been most invested, the Internet of Things can be highlighted, which allows sensing and acting in real time. In this context, identifying which data transmission technology is the most suitable for a given IoT solution is a relevant problem, since the wrong selection of data transmission technologyin an IoT project may result in an inadequate, or inefficient, solution for agribusiness, whereas there are different scenarios, with different geographic areas, different crops and seeds, varied reliefs, among several other characteristics that should be considered. This work aims to investigate the characteristics of heterogeneous wireless data transmission technologies in IoT scenarios for agribusiness, as well as to propose a web application that, given the characteristics of an IoT scenario in agribusiness, presents the best wirelessdata transmission technologies to DSS - IoT projects for Agribusiness.
\end{abstract}

Keywords: internet of things, agribusiness, wireless data transmission technologies

Data da Submissão: 29/08/2021

Data de aceitação: 16/12/2021

Chagas, C. \& Lins, F. \& Nóbrega, O. - Agroconnect - Sistema de Apoio à Tomada de Decisão de Tecnologia sem Fio de Transmissão de Dados para Soluções de IoT no Agronegócio 
Este artigo está licenciado sob forma de uma licença Creative Commons Atribuição-NãoComercial-SemDerivações 4.0 Internacional (CC BY-NC-ND 4.0) https://creativecommons.org/licenses/by-nc-nd/4.0/

\section{Introdução}

A agricultura, considerada a base da vida humana, é a principal fonte de grãos alimentícios e outras matérias-primas, papel vital no crescimento da economia do país (RAMYA et al., 2018). Contudo, o desenvolvimento da agricultura também trouxe resultados negativos ao meio ambiente, como a utilização inapropriada dos recursos naturais, água, ar e solo, prejudicando a sua qualidade e disponibilidade (NUNES, 2007). Para (GUERREROIBAÑEZ et al., 2017) estas características, associado ao aumento da população mundial, mostra que as técnicas tradicionais utilizadas na agricultura não conseguem suprir mais a demanda por insumos, necessitando de novas técnicas ou métodos que possibilitem maior eficiência e acurácia com o menor impacto na natureza.

Diante desse cenário se faz necessário uma mudança significativa para satisfazera demanda atual, criando mecanismos ou soluções que gerem equilíbrio entre a produçãoe otimização dos recursos utilizados (água, fertilizantes, entre outros) contribuindo para uma produção sustentável (GUERRERO-IBAÑEZ et al., 2017). Procurando melhores soluções para este problema, há muito tempo vem se desenvolvendo maneiras de modernizar a agricultura e torná-la mais rentável, produtiva com o menor impacto ambiental possível, otimizando a utilização dos recursos que são escassos (FERRARI; SALES, 2017).

Neste cenário, enquanto a agricultura mundial está passando pela industrialização,é importante desenvolver a informatização agrícola ao mesmo tempo. Esta informatização tornou-se a tendência de desenvolvimento para a agricultura mundial (TONGKE, 2013). Por isso, modernizá-la através de soluções tecnológicas como a Internetdas Coisas (IoT - Internet of Things) aumenta a produtividade, utilizando-se de técnicasde agricultura inteligente (Smart Agriculture, e-agricultura, agricultura intensiva) (RAMYA et al., 2018). A agricultura inteligente é descrita como um novo conceito que surgiu apóso rápido desenvolvimento das TIC (Tecnologias de Informação e Comunicação) e da Internet, considerado um campo emergente combinando os avanços em informática agrícola, desenvolvimento agrícola e empreendedorismo, para fornecer melhores serviços agrícolas, maior disseminação de tecnologia e fornecimento de informações através dos avanços nas TIC e na Internet, considerando sempre o menor impacto ambiental (GAKURU et al., 2008).

A agricultura inteligente tem o objetivo de auxiliar o agricultor sobre as condiçõesda produção através de ferramentas agrícolas inteligentes, tornando o ciclo de vida de uma colheita e as condições ambientais de cultivo são mais fáceis de rastrear em tempo real. A IoT para a agricultura pode ajudar os agricultores a rastrear o uso de água, a densidade de nutrientes e a ajustar a quantidade de fertilizantes e pesticidas utilizados. Dessa forma, os agricultores podem garantir rendimentos de colheita ideais e sistemas de gestão agrícola aprimorados (SOKOLOVA, 2021).

Estas ferramentas, conforme (RUIZ-GARCIA et al., 2009), estão entrando em uma nova fase, oferecendo vasta heterogeneidade de tecnologias de transmissão de dados, comdiferentes 223

Chagas, C. \& Lins, F. \& Nóbrega, O. - Agroconnect - Sistema de Apoio à Tomada de Decisão de Tecnologia sem Fio de Transmissão de Dados para Soluções de IoT no Agronegócio 
alcances, taxas de transmissão e consumo energético.

Desta forma, identificar qual tecnologia de transmissão de dados é a mais adequada para uma determinada soluçãode IoT é um problema relevante a ser atacado, uma vez que a seleção equivocada da tecnologia de transmissão de dados em um projeto, pode resultar em uma solução inadequada ou ineficiente de IoT para o agronegócio, visto que existem diferentes cenários, com áreas geográficas distintas, culturas e/ou cultivos variados, relevos variados, entre várias outras características que devem ser consideradas.

Através da investigação das características das tecnologias heterogêneas detransmissão de dados sem fio em cenários de IoT para o agronegócio inteligente, este trabalho tem como objetivo apresentar um sistema de apoio a tomada de decisão para a seleção de tecnologias de transmissão sem fio em projetos de IoT no agronegócio.

Este trabalho está dividido em 6 seções incluindo esta. Na Seção 2, é feita uma revisão bibliográfica sobre as tecnologias utilizadas na agricultura e os principais trabalhos relacionados com o assunto. Na Seção 3 é apresentado a proposta do sistema para classificação das tecnologias de transmissão de dados sem fio para IoT no cenário do agronegócio. As Seção 4 e 5 apresentam a implementação da proposta juntamente coma validação da mesma. Por fim, na última seção é apresentada a conclusão juntamente com os trabalhos futuros.

\section{Revisão da Literatura}

Diversas pesquisas foram concluídas no domínio da agricultura que forneceram uma maneira de coletar o status das plantações e do campo para fins de monitoramento auxiliando os usuários na tomada de decisão sobre o tratamento necessário. Redes de sensores foram usadas para detectar os atributos físicos do entorno quanto a variações e situação atual na área de irrigação, monitoramento de doenças de plantas, monitoramentode gado etc.

Na agricultura, as Tecnologias de Sensores Sem Fio (Wireless SensorTechnologies WST) devem ser capazes de operar em uma ampla gama de ambientes, como vinhas, pomares, topografias planas e complexas e em uma série de condições climáticas. Nesses cenários o levantamento das tecnologias que podem ser usadas demanda tempo e são complexos por conta de vários fatores que influenciam a qualidadedo sinal de rádio frequência (RUIZ-GARCIA et al., 2009). Com o avanço das tecnologias de IoT, é natural que sua aplicabilidade seja cada vez mais abrangente. São inúmeros os segmentos e empresas que utilizam sensores IoT para ganhar eficiência, economia de recursos e evitar erros nas suas operações (ALIGER, 2019).

O levantamento de vários cenários e as ferramentas que são utilizadas, feito por Ramya et al., (2018), descreve os benefícios de sua utilização juntamente com os cenários. As Redes de Sensores Sem Fio (Wireless Sensor Networks - WSN) são usadas em dadosde monitoramento de vídeo e fornecem maior largura de banda.

Dentre as tecnologias de WST, destacam-se as redes de sensores, que podem ser utilizadas para monitorar temperatura do ar, umidade do ar, luz do ambiente, umidade dosolo e temperatura que os ajudaram a analisar o estado atual do viveiro de plantas (ZHANG et al., 2004). Essa rede também pode ajudar a encontrar as doenças das plantas. Baggio (2005) explorou a rede de sensores para lidar com doenças da cultura da batata que se desenvolve devido ao aumento da umidade, fazendo uso de sensores para medir a umidade e a temperatura de forma a ajudá-lo a diminuí-la.

Chagas, C. \& Lins, F. \& Nóbrega, O. - Agroconnect - Sistema de Apoio à Tomada de Decisão de Tecnologia sem Fio de Transmissão de Dados para Soluções de IoT no Agronegócio 
O estudo realizado por Teixeira e Almeida (2017) implantou a tecnologia LoraWan para conexão dos dispositivos com o objetivo de diminuir o prejuízo em épocas de clima descontrolado em uma plantação de café em uma área de $2,85 \mathrm{Km}^{2}$ apresentandoum relevo bastante irregular. Foi constatado conjuntamente com o produtor agrícola que aplicando a tecnologia LoRa ${ }^{\circledR}$ em uma WSN e implementando-a nesta área, possibilitariauma previsão dos índices relevantes e de maior impacto na colheita, e consequentementeações poderiam ser tomadas com antecedência, como o maior controle do sistema de irrigação, minimizando assim os prejuízos decorrentes das intempéries (TEIXEIRA; ALMEIDA, 2017).

Em seu estudo, Hayes et al. (2005) desenvolveram um sistema que permite o monitoramento de temperatura simultâneo em dois ou mais navios de pesca usando redesde sensores sem fio personalizados operando em uma rede GSM, esse sistema permite que várias redes de sensores sejam integradas e que o usuário possa monitorar a temperatura em duas ou mais dessas redes. Neste sistema, uma rede de sensores sem fio é composta por uma estação base e vários data loggers que monitoram a temperatura. Este sistema foi testado colocando uma rede de sensores sem fio em dois locais diferentese monitorando suas temperaturas. Este sistema permite que a temperatura seja monitoradaa bordo de um ou mais navios pesqueiros simultaneamente.

O sistema de mapeamento de produção de silagem, desenvolvido por Lee et al. (2005), utiliza módulos Bluetooth para transferir informações do sensor de umidade para um computador host, porém ocorreram problemas de desconexão quando a distância entreo receptor e o transmissor ultrapassava 10 metros.

A técnica de localização proposta por Abouzar et al. 2016) é baseada em âncora, probabilística, distribuída e baseada em alcance, utiliza amostras recebidas de indicação de força de sinal (RSSI) para localização em WSN's estáticas utilizando o ZigBee. O algoritmo é chamado de modelo bayesiano para agregação de informações, e é particularmente adequado para agricultura de precisão ou aplicações com requisitos de precisão semelhantes, tamanho da rede e conectividade de nó.

A viabilidade de uma rede de sensoriamento remoto baseada em ZigBee, destinada à viticultura de precisão, mostrado por Morais et al. (2008), tinha os nós da redealimentados por baterias recarregadas com energia sustentável. Os testes dessa proposta foram divididos em duas etapas: laboratorial e em um vinhedo, realizado a calibração e validação da proposta. Para isso, um sensor MPWiNodeZ foi usado para adquirir dados de temperatura do ar e do solo, umidade relativa e radiação solar (MORAIS et al., 2008).

Em (JAWARD et al., 2018) foi proposto uma rede de sensores para agricultura baseado em ZigBee, utilizando 16 nós sensores distribuídos na área rural, conectados a 4 roteadores conectados ao roteador principal. Responsável por cobrir uma área de $50 \mathrm{~m}^{2}$, coletando informações sobre temperatura e umidade do ar e umidade do solo.

O artigo de Namani e Gonen (2020) discute sobre uma nova técnica demonitoramento do campo de cultivo com a ajuda de Drones Inteligentes, controlando-os remotamente e monitorando todos os parâmetros necessários que ajudam na previsão decampo e análise de dados. O Drone inteligente seria um veículo aéreo de asa fixa que pode transportar muitos sensores, alcançar velocidades maiores, ter maior tempo de voo e cobrir grandes áreas. Ele possui um software embarcado para planejamento e controle de voo baseado na navegação GPS (Global Positioning System), bem como no Google Maps. O modelo proposto é composto por

Chagas, C. \& Lins, F. \& Nóbrega, O. - Agroconnect - Sistema de Apoio à Tomada de Decisão de Tecnologia sem Fio de Transmissão de Dados para Soluções de IoT no Agronegócio 
3 módulos - Sensoriamento, Comunicação e Coordenação.

O Smart Drone possui modem 4G/LTE com tecnologias sem fio IoT embarcadas como Wi-Fi, ZigBee redes aéreas que ajudam na comunicação sem fio para controlá-lo remotamente e receber os dados adquiridos por ele (NAMANI; GONEN, 2020).

Com relação às técnicas de eficiência energética relacionadas às redes de sensoresno agronegócio, para a utilização mais produtiva de água, é proposto por Popli et al. (2019) superar as desvantagens dos sistemas convencionais de irrigação, como desperdíciode água (devido à percolação, evaporação), produção abreviada da cultura por meio de sensores de umidade do solo que são implantados no campo de plantio de uma fazenda. Posteriormente, os dados agregados a partir destes múltiplos sensores serão repassados para a estação base usando NBIoT (NarrowBand IoT) e, em seguida, para a nuvem NBIoT.

Os autores concluem que a capacidade de um sensor implantado na área agrícolaserá prejudicada pela interferência gerada pelos nós dos sensores adjacentes, dispersores, diferença entre terreno etc.

O trabalho apresentado por Morijo (2019) foca no desenvolvimento de uma plataforma IoT em um ambiente Fog Computing (Computação em Névoa), que inclui uma rede de dispositivos IoT interconectados por rede sem fio com tecnologias diversascomo WiFi, LoRa e ZigBee, um middleware IoT open-source ao qual foi atribuído a capacidade do tratamento individual de cada objeto sensor através da técnica de fusão dedados. Como ambiente de testes foi desenvolvida uma estação meteorológica composta de diferentes sensores, conseguiu-se uma distância aproximada de $100 \mathrm{~m}$ para ZigBee e $2 \mathrm{~km}$ utilizando-se Lora, entre o nó e o gateway com perda mínima de dados. Entende-seser suficiente para comprovar os conceitos e modelos adotados no projeto.

No Quadro 1 é apresentado um resumo dos trabalhos e suas tecnologias de conexão sem fio utilizadas, juntamente com os cenários no agronegócio. Verifica-se queos trabalhos fazem uso de diversas tecnologias de conexão sem fio, mas não apresentam ou justificam as tecnologias selecionadas para os desenvolvimentos de suas pesquisas.

Desta forma, ao analisar o Quadro 1, verifica-se que um aumento no uso dos dispositivos IoT utilizados na agricultura, resulta em uma heterogeneidade de tecnologiasde conexão sem fios para a troca de dados de sensoriamento.

Porém, nos artigos pesquisados não é apresentada a justificativa de escolha destas tecnologias, o que pode resultar em custos desnecessários, tanto financeiramente, quantona superestimação do uso de tecnologias, o que pode inviabilizar a implementação destassoluções mercadológicas em larga escala.

Diante disso, faz-se necessário uma forma de classificar qual tecnologia melhor se adequa a qual cenário do agronegócio.

Chagas, C. \& Lins, F. \& Nóbrega, O. - Agroconnect - Sistema de Apoio à Tomada de Decisão de Tecnologia sem Fio de Transmissão de Dados para Soluções de IoT no Agronegócio 
Quadro 1: Tecnologias utilizadas na Agricultura de Precisão.

\begin{tabular}{|c|c|c|c|c|}
\hline Tecnologia & Transferência & Área & Agronegócio & Artigo \\
\hline LoraWan & $\begin{array}{l}300 \text { bps a } \\
31,2 \mathrm{kpbs}\end{array}$ & $2,85 \mathrm{Km}^{2}$ & Cultivo de café & Teixeira; Almeida (2017) \\
\hline \multirow[t]{3}{*}{ ZigBee } & $\mathrm{N} / \mathrm{I}$ & $\mathrm{N} / \mathrm{I}$ & Avicultura & Morais et al.,2008 \\
\hline & $\mathrm{N} / \mathrm{I}$ & N/I & $\begin{array}{l}\text { Manejo de pragas e } \\
\text { sensoriamento de ph do solo }\end{array}$ & Abouzar et al. (2016) \\
\hline & $\mathrm{N} / \mathrm{I}$ & $50 \mathrm{~m}^{2}$ & $\begin{array}{l}\text { Coleta de umidade e } \\
\text { temperatura do ar }\end{array}$ & Jaward et al. (2018) \\
\hline Bluetooth & $\mathrm{N} / \mathrm{I}$ & $4600 \mathrm{~m}^{2}$ & $\begin{array}{l}\text { Mapeamento de rendimento da } \\
\text { Silagem }\end{array}$ & Lee et al.(2005) \\
\hline GPRS & $433 \mathrm{MHz}$ & $\mathrm{N} / \mathrm{I}$ & Navios de pesca & Hayes et al. (2005) \\
\hline $\begin{array}{l}\text { WiFi, LoRa e } \\
\text { ZigBee }\end{array}$ & $\begin{array}{l}0,018 \text { à } 37,5 \\
\text { Kbps ( ESP32) }\end{array}$ & 208.mil m² & plantação de soja, milhoe trigo & Morijo (2019) \\
\hline NBIoT & & & Irrigação & Popli et al. (2019) \\
\hline $\begin{array}{l}\text { 4G/LTE, } \\
\text { Wi-Fi } \\
\text { e ZigBee }\end{array}$ & $\mathrm{N} / \mathrm{I}$ & $\mathrm{N} / \mathrm{I}$ & $\begin{array}{l}\text { monitoramento aéreo dasáreas } \\
\text { agrícolas }\end{array}$ & Namani; Gonen (2020) \\
\hline
\end{tabular}

\section{AgroConnect - Proposta de Sistema de Apoio à tomada de decisão de Tecnologias sem fio de transmissão de dados para IoT no agronegócio}

Este trabalho apresenta o Agroconnect, solução que tem como objetivo auxiliar na tomada de decisão de tecnologias de transmissão sem fio de dados em cenários de IoTno agronegócio. A solução proposta é composta por 3 módulos como ilustra a Figura 01.O MÓDULO DE ENTRADA DE DADOS tem como objetivo possibilitar a inserção, validação e normatização das características dos dados referentes ao cenário de agronegócio a ser planejado pelo usuário. O MÓDULO DE ARMAZENAMENTO possibilita que as características que definem as tecnologias de transmissão de dados sejam armazenadas para cálculos futuros. Por fim, o MÓDULO DE CLASSIFICAÇÃO E SELEÇÃO tem como função a classificação das tecnologias de transmissão de dados, indicando uma lista de tecnologias adequadas para o cenário planejado.

Chagas, C. \& Lins, F. \& Nóbrega, O. - Agroconnect - Sistema de Apoio à Tomada de Decisão de Tecnologia sem Fio de Transmissão de Dados para Soluções de IoT no Agronegócio 
Figura 01: Visão Geral dos Módulos do Sistema.

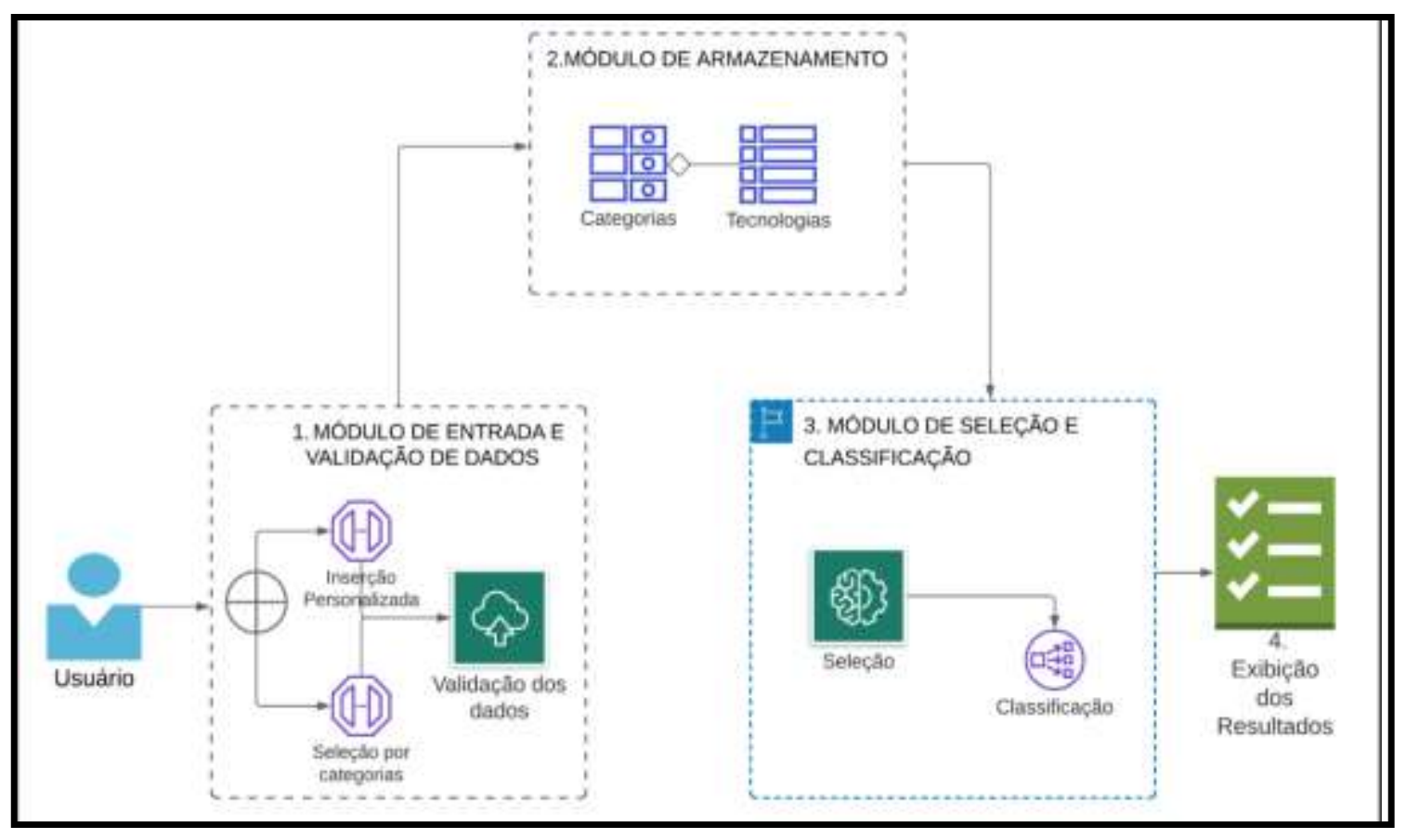

Fonte: Autores (2021).

Nas próximas subseções será explicado em detalhes as funcionalidades de cada umdos módulos envolvidos na proposta.

\subsection{Módulo de entrada e validação de dados}

Sabe-se que cada tecnologia de transmissão de dados possui diversas características que a define em contraparte com a heterogeneidade de um cenário agrícola.Desta forma, foi criada uma relação com as características que definem os cenários agrícolas e as que definem as tecnologias, selecionando as variáveis que atendem os seguintes critérios:

- Distância entre o nó (dispositivo IoT sem fio) e o ponto de acesso à internet (gateway)mais próximo;

- Taxa de transferência de dados;

- Tipo de fonte energética dos dispositivos IoT que será usado no projeto (usado comocritério de ordenação).

Nesse módulo é possível escolher duas formas distintas de entrada de dados:

- Inserção personalizada;

- Seleção por categorias.

Na inserção personalizada o usuário insere manualmente os dados desejados para:

- Menor distância $\{d\}$ entre o nó/controlador de transmissão e o gateway de acessoà Internet,

- Taxa de transferência $\{t x\}$ mínima necessária,

Chagas, C. \& Lins, F. \& Nóbrega, O. - Agroconnect - Sistema de Apoio à Tomada de Decisão de Tecnologia sem Fio de Transmissão de Dados para Soluções de IoT no Agronegócio 
- Tipo de fonte energética $\{\varepsilon\}$ utilizada pelo dispositivo IoT.

Uma vez inseridas estas informações, é verificado se as entradas são válidas, ou seja, $\{d, t x \in N A(d, t x)>0\}$. Uma vez que estas informações estejam válidas, estas são enviadas para o MÓDULO DE ARMAZENAMENTO para futura classificação.

Caso o usuário não disponha destas informações para inserção personalizada, uma segunda possibilidade é a seleção por categorias de tecnologias já classificadas, onde é proposto ao usuário o agrupamento das tecnologias em intervalos para cada característica definida disponíveis no MÓDULO DE ARMAZENAMENTO, como apresentado no Quadro 02, facilitando assim a entrada de dados.

O agrupamento dessas tecnologias acontece seguindo a seguinte regra: dado o valor $x$ para uma característica presente no Quadro 2, $x$ pertencerá à categoria $C$ se o valor $x$ estiver entre o intervalo definido para a categoria, ou seja,

$$
\{x \in C \mid \text { início_do_intervalo } \leq x \leq \text { final_do_intervalo }\} .
$$

Essa regra aplica-se tantopara a distância $\{D\}$ quanto para a taxa de transferência $\{T x\}$.

Uma vez finalizada a seleção de intervalos, estas informações são tratadas na próxima etapa apresentada na subseção a seguir

\subsection{Módulo de armazenamento}

Após a pesquisa bibliográfica para verificar as tecnologias IoT mais utilizadas nocenário do agronegócio, juntamente com as características que as definem, foi selecionado das especificações as características necessárias para definições mínimas decenários, tendo sua definição conforme abaixo:

- $\{D\}$ - Distância: distância máxima que pode ser alcançada conforme especificação;

- $\{T \mathrm{x}\}$ - Taxa de transferência: taxa alcançada para transferir os dados;

- $\{E\}$ - Consumo Energético: energia consumida ao transmitir os dados.

Essas características são utilizadas para classificar quais serão as tecnologias que melhor se adequam ao cenário recebido como entrada. No Quadro 02 são apresentadas as tecnologias e suas características que serão utilizadas no MÓDULO DE ARMAZENAMENTO para classificá-las de acordo com os dados de entrada do usuário.

Chagas, C. \& Lins, F. \& Nóbrega, O. - Agroconnect - Sistema de Apoio à Tomada de Decisão de Tecnologia sem Fio de Transmissão de Dados para Soluções de IoT no Agronegócio 
Quadro 2: Tecnologias utilizadas na Agricultura de Precisão.

\begin{tabular}{|c|c|c|c|}
\hline Nome & $E(\mathrm{dBm})$ & $T x$ (mbpps) & $D$ (metros) \\
\hline Zigbee Global & 0 & 0.25 & 10 \\
\hline Bluetooth Classe 1 & 20 & 3 & 100 \\
\hline Bluetooth Classe 2 & 4 & 2 & 10 \\
\hline Bluetooth Classe 3 & 0 & 1 & 1 \\
\hline BLE Classe 1 & 10 & 2 & 50 \\
\hline BLE Classe 1.5 & -20 & 1 & 50 \\
\hline BLE Classe 2 & -20 & 0.5 & 50 \\
\hline BLE Classe 3 & -20 & 0.125 & 50 \\
\hline WIFI 2.4Ghz & 100 & 54 & 100 \\
\hline WIFI 5Ghz & 50 & 1024 & 100 \\
\hline LoraWAN & 20 & 0.05 & 15000 \\
\hline GPRS & 33 & 2.5 & 10000 \\
\hline $4 \mathrm{G}$ & 45 & 300 & 10000 \\
\hline WirelessHART & 10 & 0.25 & 200 \\
\hline Wibree & 10 & 1 & 10 \\
\hline Narrow Band & 20 & 0.127 & 20000 \\
\hline Sigfox & 22 & 0.0006 & 40000 \\
\hline
\end{tabular}

\subsection{Módulo de seleção e classificação}

Nesta fase, inicialmente é necessário, dado um conjunto de tecnologias heterogêneas, selecionar um conjunto que atenda os requisitos mínimos do projeto de IoT.Como entrada deste módulo, caso seja escolhida a seleção por categorias, é recebida umalista de categorias $L_{C}=$ $\left\{C_{1}, C_{2}, \ldots, C_{n}\right\}$ e suas respectivas tecnologias de transmissão de dados $\left\{T_{1} \ldots T_{n}\right\}$. Logo, as tecnologias de transmissão de dados $T_{1 \ldots n}$ retornadas são a interseção entre as tecnologias presentes na categoria selecionada para a taxa de transferência $C_{T x}$ e para a distância $C_{D}$, desta forma tem-se que $T_{1 . . n}=C_{T x} \cap C_{D}$. Caso seja escolhida a inserção personalizada, são selecionadas as tecnologias de transmissão de dados que atendem à lista L conforme equação 1 : 


\section{RMP}

$$
L=\left\{T_{1}, \ldots, T_{\mathrm{n}}\right\} \mid T_{1 \rightarrow \mathrm{n}} \in L \Leftrightarrow[(d \leq D) \mathrm{A}(t x \leq T x)]
$$

Como resultados, são retornadas as 5 primeiras tecnologias de transmissão de dados, ordenados pela distância e taxa de transferência de dados, caso a fonte de energiaselecionada seja igual à "energia elétrica". caso seja diferente, é ordenado pelo consumo energético, distância de forma crescente que mais se aproximam das necessidades do cenário de agronegócio.

Em um segundo momento, uma vez identificada a lista de cinco tecnologias que atendem aos requisitos mínimos do projeto de IoT, é utilizado um algoritmo de classificação para marcar a tecnologia que mais se aproxima do cenário pretendido pelo usuário. Desta forma, utilizou-se o algoritmo de classificação K-NN (K-Nearest Neighbors) (Norvig, et al., 2013), onde o aprendizado é baseado "na proximidade" entreum dado de entrada (um vetor) e outro em uma base de dados, que resulte a melhor tecnologia dentre as tecnologias selecionadas. Para isto utilizou-se a distância euclidiana, possibilitando indicação da tecnologia de transmissão (Tn) de dados que mais se aproxima do cenário IoT projetado para o agronegócio pela fórmula:

$$
T_{n}=\sqrt{ } 0,8 \cdot\left((T x-t x)^{2}\right)+0,2 \cdot\left((D-d)^{2}\right.
$$

Como pode ser visto na equação 4 , a taxa de transferência tem um peso 4 vezes maior que a distância, pois os resultados experimentais mostraram que a distância estavatendo uma influência mais significativa no resultado. Com isso, foi ajustado o peso para a taxa de transferência para que o mesmo pudesse ter uma relevância maior no resultado.

Quanto à variável do consumo energético $\{\mathrm{E}\}$, caso a solução de IoT utilize fontede energia elétrica convencional, esta variável não será considerada, pois não influenciarána eficiência da transmissão das placas de redes sem fio. Porém, se utilizar painéis fotovoltaicos, esta variável é considerada por influenciar na decisão da tecnologia de transmissão sem fio.

Como resultado é apresentado uma lista de tecnologias de transmissão sem fio ordenada por prioridades de escolha, para o cenário projeto de IoT no Agro 4.0 informadopelo usuário.

\subsection{Fluxograma simplificado}

O fluxograma apresentado na Figura 02 descreve o fluxo apresentado nas seções anteriores, desde a seleção do método e entrada dos dados, validação dos dados, filtro e classificação até a saída dos resultados. Pode-se notar a forma como o método de escolhade dados influencia na escolha das Tecnologias de transmissão de dados. 
Figura 02: Fluxo da proposta de seleção de Tecnologias de transmissão de dados.

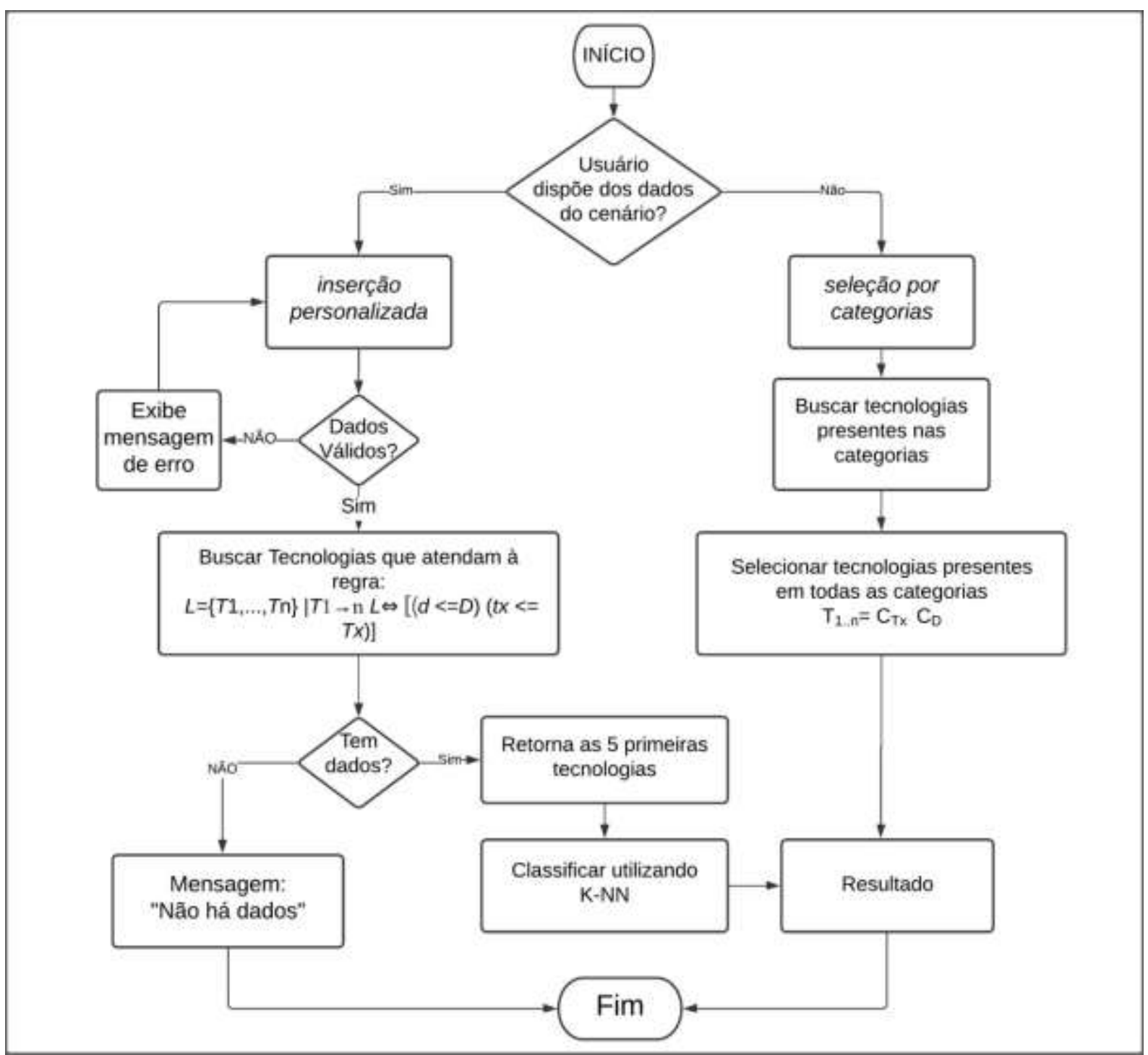

\section{Implementação do Agroconnect}

Para validar a proposta desenvolveu-se um sistema utilizando o framework React JS para construir a interface do usuário, Java versão 11 onde será implementadoas regras e PostgreSQL para armazenar as características referente às tecnologias.

Na Figura 05 observa-se a implementação da interface para os módulos apresentados na seção 3 onde é possível cadastrar novas tecnologias, escolher qual tipo de entrada de dados e possibilita verificar as características de cada tecnologia cadastrada,como pode ser visto na Figura 05.

Chagas, C. \& Lins, F. \& Nóbrega, O. - Agroconnect - Sistema de Apoio à Tomada de Decisão de Tecnologia sem Fio de Transmissão de Dados para Soluções de IoT no Agronegócio 
Figura 05: Escolha da entrada dos dados para Seleção das Tecnologias.

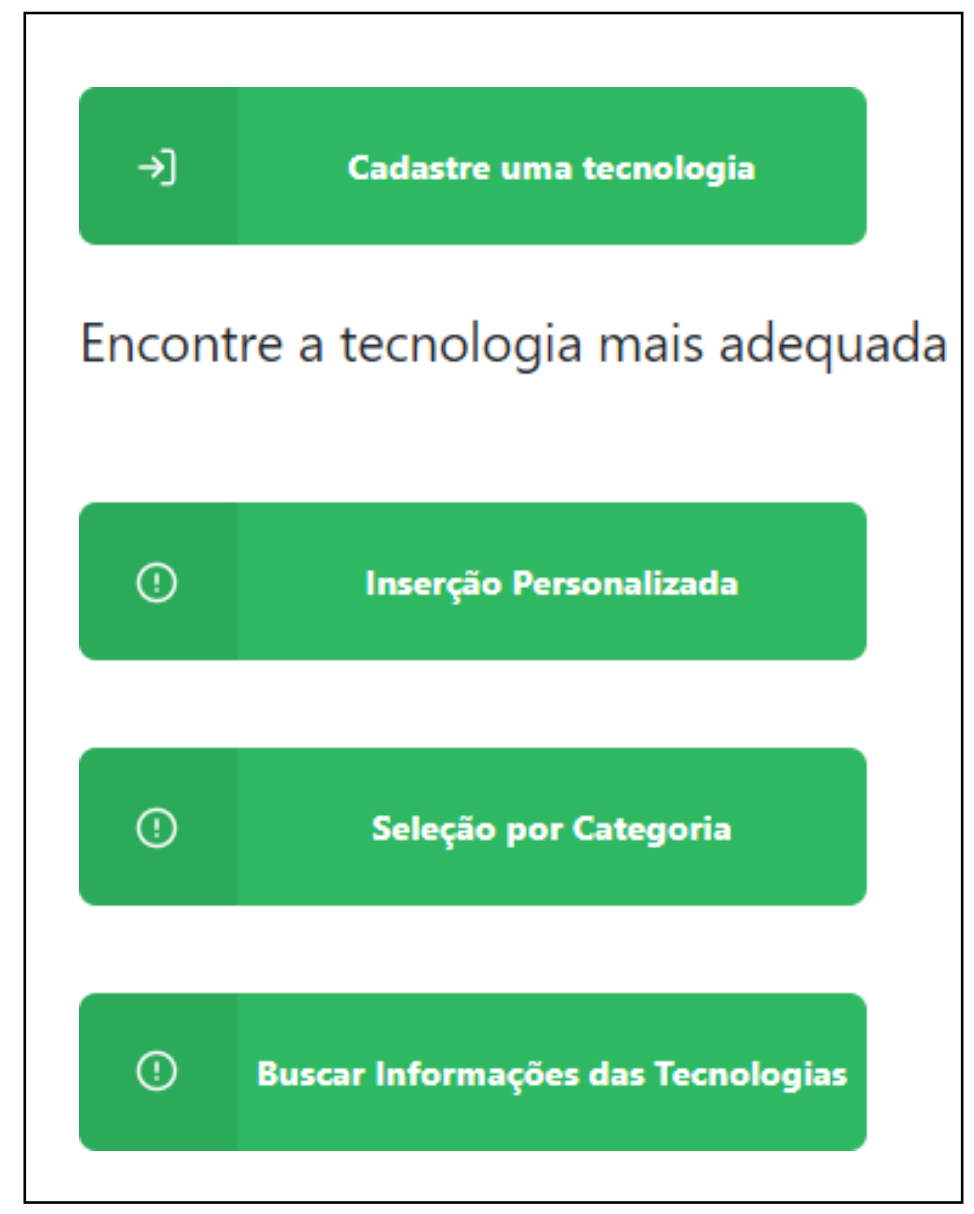

Para a implementação da seleção por categoria as tecnologias apresentadas no Quadro 2 foram agrupadas da seguinte forma:

- Distância: [0, 10], $(10,100],(100, \infty)$

- Taxa de transferência de dados: $[0,1),[1,2),[2,3],[54,54]$

Onde, "(" ou ")" o dado não está incluído no intervalo (intervalo aberto) e "[" ou"]" o dado está incluído no intervalo (intervalo fechado).

$\mathrm{Na}$ inserção personalizada além da entrada dos dados pelo usuário é possível escolher o tipo de fonte energética entre:

\section{- [Bateria, Energia Elétrica, Energia Solar]}

Na Figura 06 tem-se o resultado da busca das tecnologias de transmissão de dadosde forma crescente que mais se aproximam das necessidades do cenário do agronegócio apresentado abaixo, destacando-se a que se acredita ser a mais adequada ao cenário utilizado. Para o exemplo abaixo foi utilizado o seguinte cenário como entrada:

Chagas, C. \& Lins, F. \& Nóbrega, O. - Agroconnect - Sistema de Apoio à Tomada de Decisão de Tecnologia sem Fio de Transmissão de Dados para Soluções de IoT no Agronegócio 


$$
\{d=2, \varepsilon=\text { "bateria" e } t x=2\}
$$

Figura 06: Resultado da Seleção de Tecnologias Sem Fio de Transmissão de Dados.

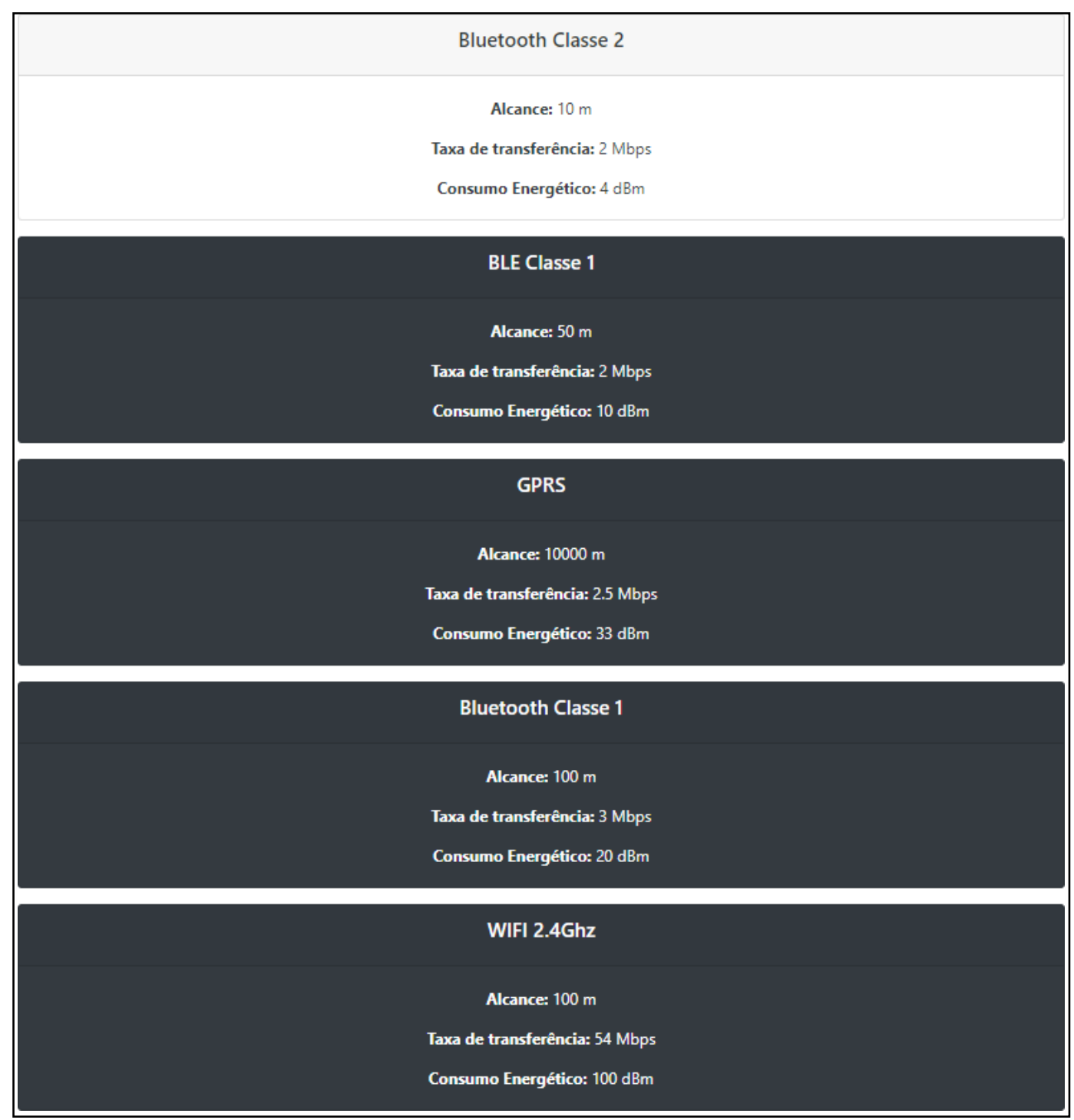

Na próxima seção usaremos a implementação apresentada para avaliação daproposta de seleção de tecnologias de transmissão de dados sem fio.

\section{Avaliação}

Para avaliação da proposta foi analisado os artigos presentes no Quadro 1, onde são levantados vários cenários com as tecnologias envolvidas e as respectivas características de seus cenários.

Como pode-se ver em [Teixeira and Almeida, 2017], foi utilizado 5 nós LoraWane 1 gateway para cobrir uma área de $2850 \mathrm{~m}^{2}$. Para facilitar a área que cada nó irá cobrirserá

$$
234
$$

Chagas, C. \& Lins, F. \& Nóbrega, O. - Agroconnect - Sistema de Apoio à Tomada de Decisão de Tecnologia sem Fio de Transmissão de Dados para Soluções de IoT no Agronegócio 
dividido a área total por 5, ficando cada nó com uma área total conforme abaixo:

$$
\text { área nó }=2850 / 5=570 \mathrm{~m}^{2}
$$

A taxa de transferência dos sensores utilizados no artigo pode chegar até a 31,2kpbs, ransformando para Mbps, medida utilizada na implementação, tem-se aproximadamente: $0.031 \mathrm{Mbps}$. Utilizando o cenário passado em nosso sistema:

$\{d=570, \varepsilon=$ "energia elétrica" e $t x=0.031\}$, temos o cenário apresentado na figura abaixo.

Figura 07: Resultado da busca.

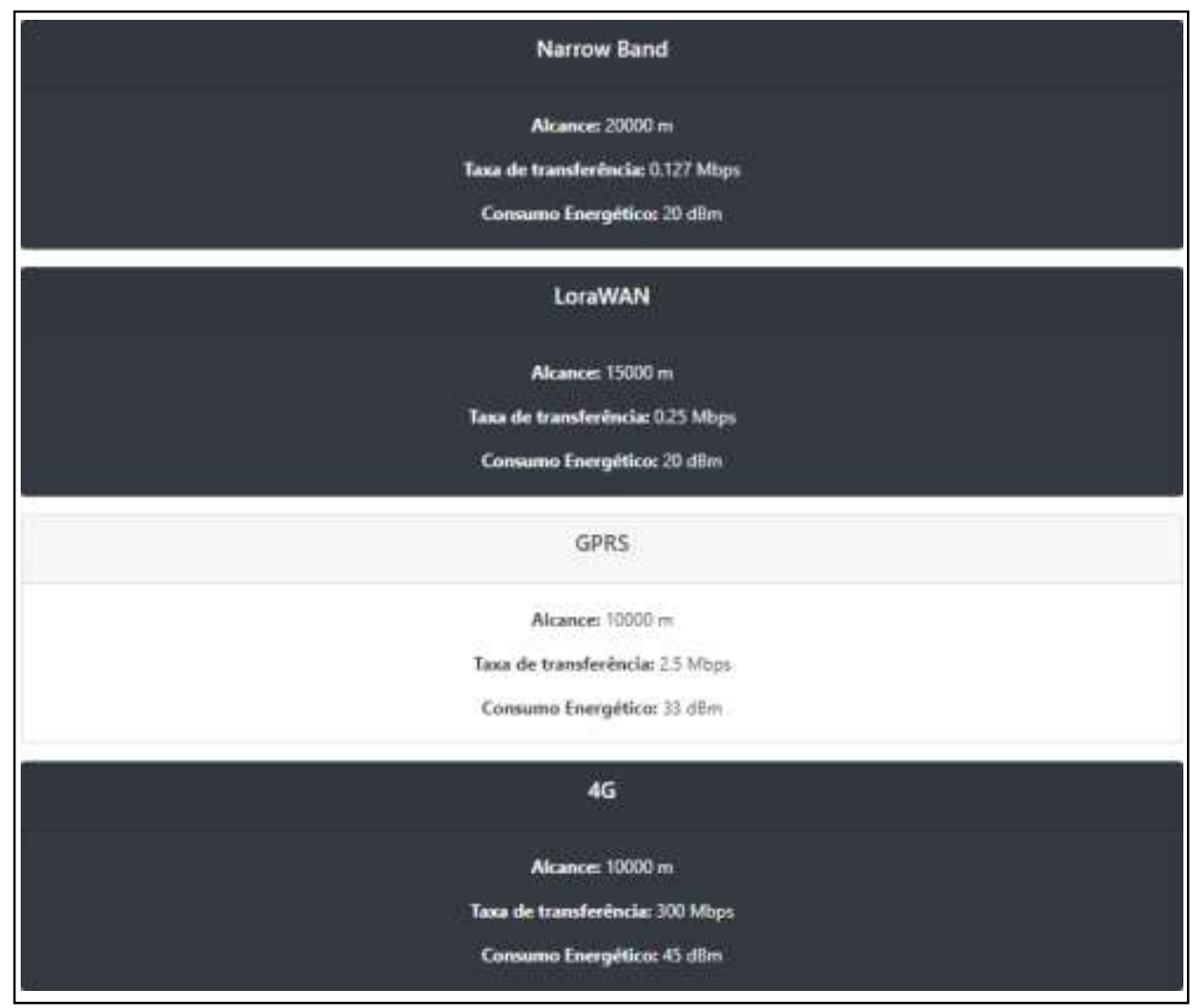

Verifica-se, assim, como Teixeira e Almeida (2017) explica em seu artigo, quemesmo existindo outras tecnologias que poderiam atender o cenário ao qual se propôs desenvolver, ao utilizar o AgroConnect obteve-se resultado semelhante ao proposto pelosautores. O Sistema implementado aponta como melhor tecnologia a ser utilizada no cenário do autor seria o GPRS por estar mais próximo da distância pretendida e ter uma taxa de transferência mais elevada.

Já Morijo (2019) implementou um sistema de monitoramento meteorológico utilizando ZigBee e LoraWan para transferência dos dados entre nós e gateway. Para o rádio utilizando Zigbee tivemos o seguinte cenário:

Chagas, C. \& Lins, F. \& Nóbrega, O. - Agroconnect - Sistema de Apoio à Tomada de Decisão de Tecnologia sem Fio de Transmissão de Dados para Soluções de IoT no Agronegócio 
Figura 08: Resultado da busca usando os dados de entrada: $\{d=363.55, \varepsilon=$ "energia elétrica" e $t x=0,05\}$.

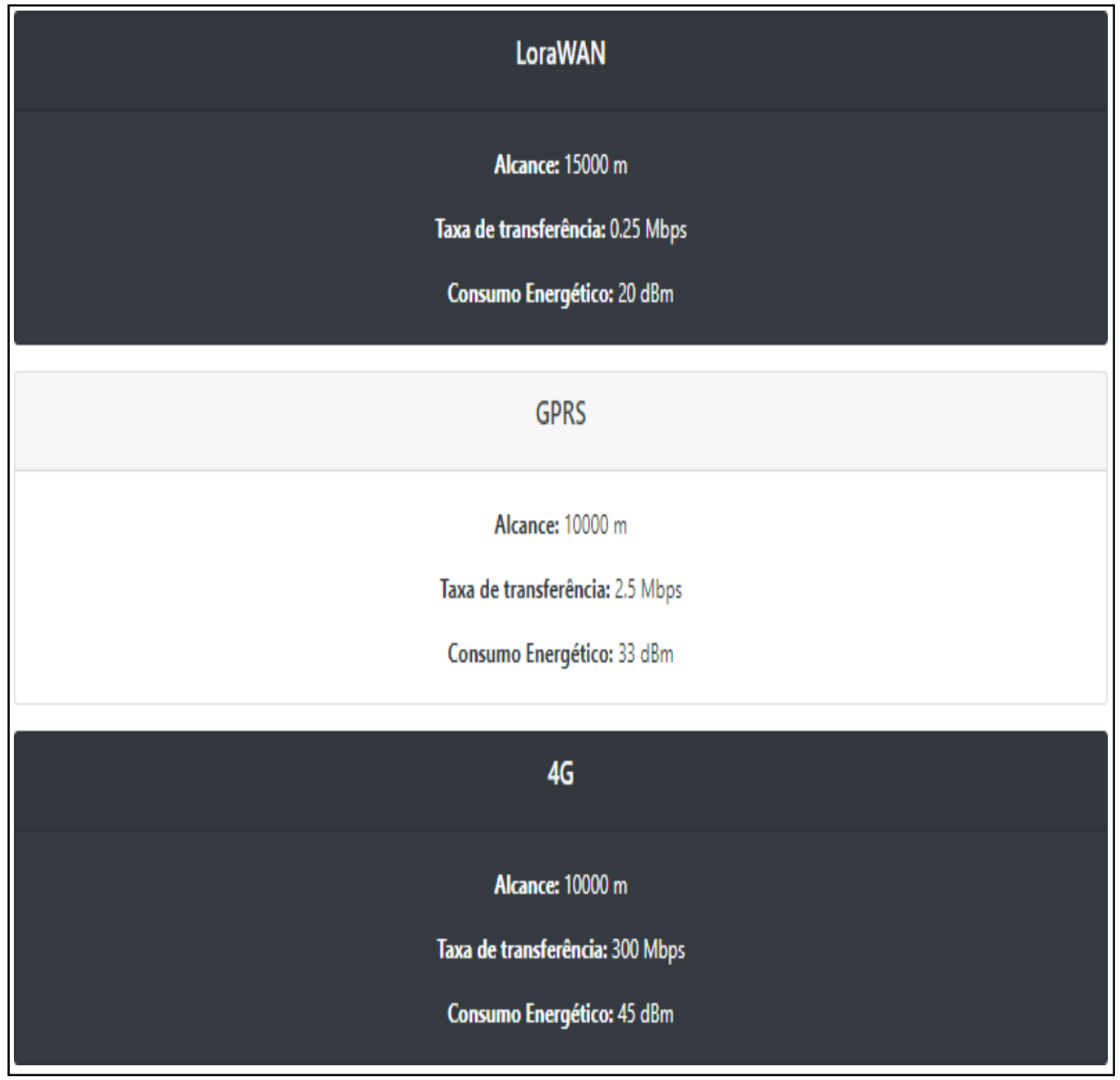

$\{d=363.55, \varepsilon=$ "energia elétrica" e $t x=N / 1\}$, como a taxa de transferência não foi informada utilizaremos a padrão da tecnologia ZigBee: $T x=0.05 \mathrm{Mbps}$.

Como utilizou-se a configuração Global do ZigBee com frequência de $2.4 \mathrm{Ghz}$ não é possível alcançar a distância de $363,55 \mathrm{~m}$ no artigo de [Morijo, 2019]. Mas, como o no artigo apresentado também faz uso do LoraWan, onde tem-se a seguinte configuração:

$\{d=2000, \varepsilon=$ "energia elétrica" e $t x=N / I\}$, como a taxa de transferência não foi informada utilizou-se a padrão da tecnologia LoraWan $T x=0.25 \mathrm{Mbps}$

Chagas, C. \& Lins, F. \& Nóbrega, O. - Agroconnect - Sistema de Apoio à Tomada de Decisão de Tecnologia sem Fio de Transmissão de Dados para Soluções de IoT no Agronegócio 
Figura 09: Resultado da busca usando os dados de entrada: $\{d=2000, \varepsilon=$ "energia elétrica" e $t x=0,25\}$.

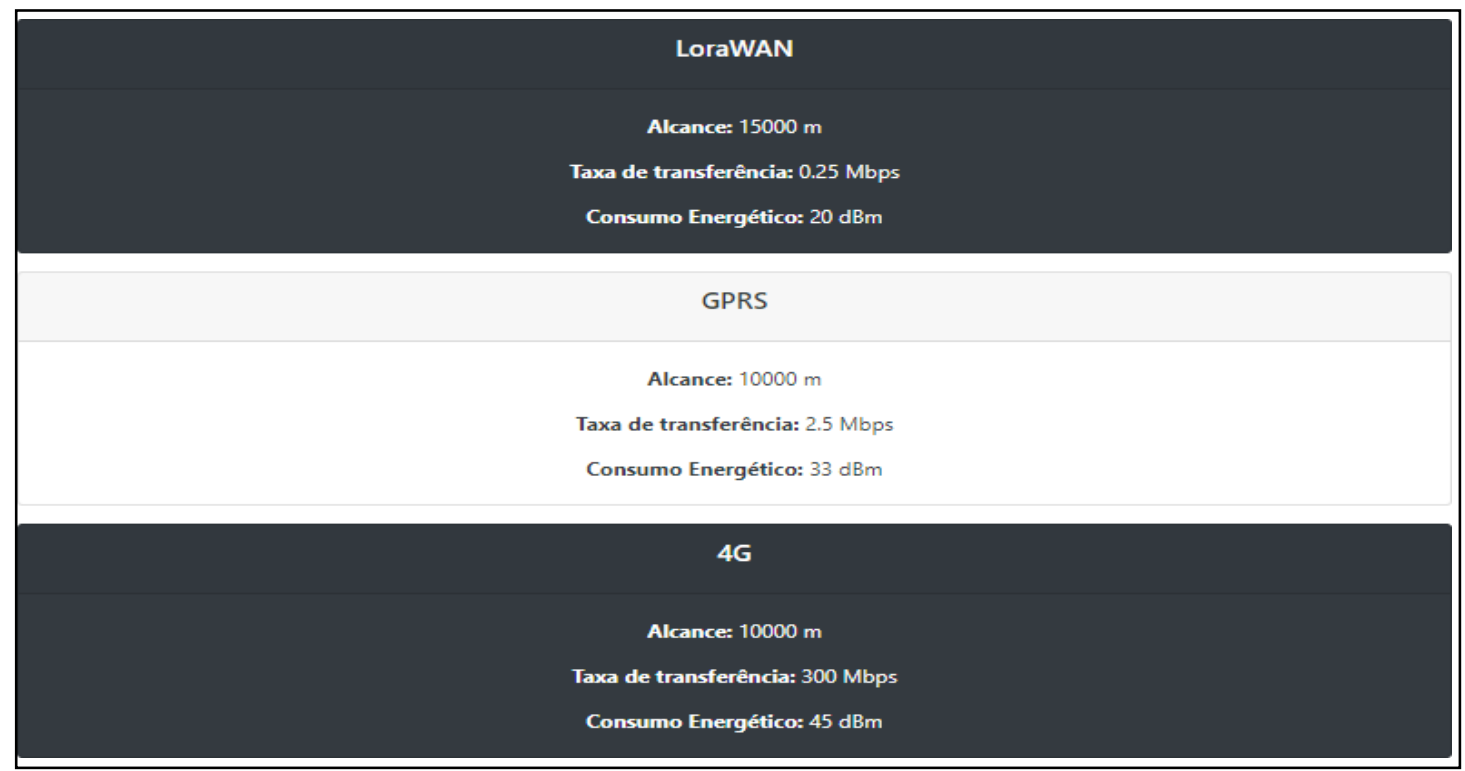

Desta forma, pode-se observar que nas análises relacionadas às figuras 08 e 09 o resultado apresentado pelo Agroconnect se mantém, indicando uma adequação aoscenários avaliados independentemente da distância entre o nó e o gateway, o quedemonstra que os resultados da solução estão de acordo com os projetos já executados. Porém, pode-se também verificar as soluções utilizadas por Mojiro (2016) poderia ter uma melhor eficiência em seu projeto se o mesmo optasse por GPRS, por ter um alcanceperto do esperado e estar utilizando uma energia elétrica como fonte energética, conforme o resultado apresentado pelo AgroConnect.

\section{Conclusão}

Com o avanço das tecnologias de IoT, é natural que sua aplicabilidade seja cada vez mais abrangente. São inúmeros os segmentos e empresas que utilizam sensores IoT para ganhar eficiência, economia de recursos e evitar erros nas suas operações. Esse aumento no uso dos dispositivos IoT utilizados na agricultura resultou em uma variedadede tecnologias de conexão sem fios utilizadas para realizar as comunicações entre os mesmos.

Devido a diversas características heterogêneas na área do agronegócio, existe a relevante necessidade de investigar e compreender quais tecnologias de transmissão de dados sem fio são utilizadas nas mais diversas soluções de IoT para o agronegócio, assimcomo, as suas principais características de transmissão que impactarão diretamente em futuras tomadas de decisão para escolha de novas soluções com o uso de IoT.

Neste trabalho foi apresentado a proposta de um sistema que tem por objetivo auxiliar na escolha de tecnologia de transmissão sem fio de dados que se adequa ao cenário de IoT no agronegócio, assim como sua implementação de forma a validá-la. O sistema tem a capacidade de abstrair a complexidade de seleção de tecnologias de acordocom o cenário escolhido.

Chagas, C. \& Lins, F. \& Nóbrega, O. - Agroconnect - Sistema de Apoio à Tomada de Decisão de Tecnologia sem Fio de Transmissão de Dados para Soluções de IoT no Agronegócio 
Este sistema permite a seleção de tecnologia de duas formas, dependendo do nívelde detalhes da especificação dos cenários, além de permitir a inserção de novas tecnologias. Caso o usuário não possua muitos detalhes do cenário é possível fazer a seleção por categorias de forma a simplificar a seleção, caso o mesmo possua todos os detalhes é possível fazer a seleção através da inserção personalizada, onde o usuário irá entrar com os valores pretendidos. Como resultado é apresentado uma lista de tecnologiasde transmissão sem fio ordenada por prioridades de escolha, para o cenário projeto de IoTno Agro 4.0 informado pelo usuário. Acredita-se que a proposta possa servir como uma ferramenta de suporte à tomada de decisão tecnológica para facilitar o desenvolvimento de projetos de IoT de forma mais eficiente considerando os cenários heterogêneos brasileiros.

Como trabalho futuro, pretende-se ampliar a quantidade de características utilizadas no cálculo de escolha da tecnologia de transmissão sem fio. Pode-se ser utilizado o consumo energético de forma mais eficiente, ao invés de ser apenas parâmetrode ordenação, largura de banda possibilitando ter uma ferramenta que considere oconsumo energético de forma mais eficiente.

\section{Referências}

ABOUZAR, P., MICHELSON, D. G., AND HAMDI, M Rssi-Based Distributed SelfLocalization for Wireless Sensor Networks Used in Precision Agriculture. IEEE Transactions on Wireless Communications, v. 15, n. 10, p. 6638- 6650, 2016.

ALIGER Os Sensores Iot E Suas Aplicações na Agricultura e Pecuária. Retrieved from: https://www.aliger.com.br/blog/conheca-os-sensores-iot-e- sua-aplicabilidade-na-agriculturae-pecuaria. 2019.

BAGGIO, A. Wireless Sensor Networks in Precision Agriculture. In ACM Workshop on Real-World Wireless Sensor Networks (REALWSN 2005), Stockholm, Sweden (Vol. 20, pp. 1567-1576). 2005.

FERRARI, A. AND SALES, G. G. R. Smart Farming: Análise bibliométrica sobre o tema. Universidade Estadual de Campinas, Faculdade Aplicada de Ciências Aplicadas. 2017.

GAKURU, M., WINTERS, K., AND STEPMAN, F. Innovative Farme. 2008. Advisory Services using Ict. In W3C Workshop "Africa Perspective on the Role of Mobile Technologies in Fostering Social Development”, Maputo, Mozambique (pp. 1- 2). 2008.

GUERRERO-IBAÑEZ, J. A. Sgreenh-Iot: Plataforma Iot para Agricultura de Precisión. Sistemas, Cibernética E Informática, 14(2). 2017.

HAYES, J., CROWLEY, K., AND DIAMOND, D. Simultaneous Web-Based Real-Time Temperature Monitoring Using Multiple Wireless Sensor Networks. In SENSORS, 2005 IEEE, pages 4 pp. 2005.

HEBEL, M. A. Meeting Wide-Area Agricultural Data Acquisition and Control Challenges Through Zigbee Wireless Network Technology. In Computers inAgriculture and Natural Resources, 23-25 July 2006, Orlando Florida. American Societyof Agricultural and Biological Engineers. 2006;

MORIJO, J. P. D. S. Arquitetura Multissensorial em Fog Computing para Dispositivos Iot com Foco em Agricultura De Precisão. 2019 
LEE, W. S., SCHUELLER, J. K., \& BURKS, T. F. Wagon-Based Silage Yield Mapping System. Agricultural Engineering International: CIGR Journal. 2005.

MORAIS, R., FERNANDES. A Zigbee Multi-Powered Wireless Acquisition Device for Remote Sensing Applications in Precision Viticulture. Computers and Electronics in Agriculture, 62(2): 94-106. 2008.

NAMANI, S., GONEN, B. Smart Agriculture Based on Iot and Cloud Computing. In 2020 3rd International Conference on Information and ComputerTechnologies (ICICT) (pp. 553556). IEEE. 2020

NORVIG, PETER RUSSEL, STUART. Modelo do vizinho mais próximo. Inteligência Artificial. Rio de Janeiro: Elsevier Editora Ltds, 2013, Vol. 3, p.1152. 2013.

NUNES, S. P. (2007). O Desenvolvimento Da Agricultura Brasileira e Mundial e a Idéia de Desenvolvimento Rural. Departamento de Estudos Socio-Econômicos Rurais. Boletim Eletrônico, Conjuntura Agricola, (157).

POPLI, S., JHA, R. K. JAIN, S. A Survey on Energy Efficient Narrowband Internet of Things (Nbiot): Architecture, Application and Challenges. IEEE Access, 7, 16739-16776. 2018.

RAMYA, B., T, T., J, T., AND ANITA, E. A. M. A Survey on Smart Agriculture Using Internet of Things. Internationa Journal of Engineering Research \& Technology (IJERT) NCICCT - Volume 6 - Issue 3. 2018

RUIZ-GARCIA, L., et al.. A review of wireless sensor technologies andapplications in agriculture and food industry: State of the art and current trends. Sensors, 9 (6): 4728 4750. 2009.

SOKOLOVA, LARA. In: O que saber sobre agricultura inteligente usando IoT:Como a tecnologia está transformando a produção de alimentos e os desafios de suaimplementação e segurança dos sistemas. Forbes Agro, 24 set. 2021.

TEIXEIRA, G. B., \& ALMEIDA, J. V. P. D. Rede LoRa ${ }^{\circledR}$ e protocolo LoRaWAN ${ }^{\circledR}$ aplicados na agricultura de precisão no Brasil, (Bachelor's thesis, Universidade Tecnológica Federal do Paraná). 2017

TONGKE, FAN (2013). Smart agriculture based on Cloud Computing and IOT.Journal of Convergence Information Technology, v. 8, n. 2, p. 210-216. 2013

Zhang, W., Kantor, G., Singh, S. Integrated Wireless Sensor/Actuator Networks in an Agricultural Application. In Proceedings of the 2ndinternational conference on Embedded networked sensor systems (pp. 317-317).2004. 